\title{
Multi-Modality Phantom Development
}

\author{
J.S. Huber, Member, IEEE, Q. Peng, and W.W. Moses, Fellow, IEEE
}

\begin{abstract}
Multi-modality imaging has an increasing role in the diagnosis and treatment of a large number of diseases, particularly if both functional and anatomical information are acquired and accurately co-registered. Hence, there is a resulting need for multi-modality phantoms in order to validate image co-registration and calibrate the imaging systems. We present our PET-ultrasound phantom development, including PET and ultrasound images of a simple prostate phantom. We use agar and gelatin mixed with a radioactive solution. We also present our development of custom multi-modality phantoms that are compatible with PET, transrectal ultrasound (TRUS), MRI and CT imaging. We describe both our selection of tissue mimicking materials and phantom construction procedures. These custom PET-TRUS-CT-MRI prostate phantoms use agargelatin radioactive mixtures with additional contrast agents and preservatives. We show multi-modality images of these custom prostate phantoms, as well as discuss phantom construction alternatives. Although we are currently focused on prostate imaging, this phantom development is applicable to many multi-modality imaging applications.
\end{abstract}

Index Terms-Positron Emission Tomography, Ultrasound, Magnetic Resonance Imaging, X-ray Computed Tomography

\section{INTRODUCTION}

$\mathrm{M}$ ulti-modality imaging plays an increasingly important role in the diagnosis and treatment of a large number of diseases. Combining modalities that provide both functional and structural information is particularly important. For instance, functional information can be acquired with single photon emission computed tomography (SPECT), positron emission tomography (PET) or functional magnetic resonance imaging (fMRI), whereas anatomical information can be acquired using x-ray computed tomography (CT), ultrasound (US) or magnetic resonance imaging (MRI).

Combining PET and CT has recently revolutionized the role of imaging in diagnosis and treatment planning for many kinds of cancer. As a result, there are commercially available PET-CT phantoms that are used for acceptance testing and routine quality evaluation of the PET-CT systems. For instance, these phantoms are used to determine how accurately the two image sets are aligned and how accurately the CTbased PET attenuation correction works. However, PET-CT imaging is not the preferred modality combination for all diseases or even all cancers. For instance, ultrasound imaging is an integral part of diagnosis and treatment procedures for many diseases, such as prostate cancer. Transrectal ultrasound (TRUS) provides good anatomical detail of the prostate region and accurately measures the prostate volume, whereas CT has poor contrast for soft-tissue (like the prostate) and it over-

Manuscript received March 20, 2009. This work was supported in part by the Director, Office of Science, Office of Biological and Environmental Research, Medical Science Division of the U.S. Department of Energy under Contract No. DE-AC02-05CH11231, and in part by Department of Defense grant number W81XWH-07-1-0020.

J.S. Huber, Q. Peng, and W.W. Moses are with Lawrence Berkeley Nationial Laboratory, Berkeley, CA 94720 USA (e-mail: jshuber@lbl.gov). estimates prostate volume. Hence, phantoms for multimodality imaging with ultrasound and PET or MRI are needed. Ultrasound phantoms are readily available commercially, but we are not aware of any commercially available US-PET or US-MRI phantoms.

We believe that imaging the prostate with ${ }^{11} \mathrm{C}$-choline using a dual PET-TRUS system will help locate cancer within the prostate region. We envision that dual PET-TRUS prostate imaging could be used to guide biopsy, guide treatment procedures, and detect local recurrence earlier than is currently possible. Therefore, we are developing a dual imaging system that acquires PET and TRUS data sets during the same patient imaging session, using methods that allow us to accurately determine the 3D location of the TRUS probe tip relative to the PET scanner. As a result, co-registration of the PET and TRUS patient images will be simple and accurate. However, prior to our patient studies, we must validate our ability to image and accurately co-register PET and TRUS images using a PET-TRUS prostate phantom.

Since the phantom that we need is not commercially available, we have built a custom PET-TRUS prostate phantom with structures that simulate the acoustical properties for TRUS and $511 \mathrm{keV}$ activity concentrations for PET. In addition, we have built custom PET-TRUS-CT-MRI phantoms with structures that also simulate the nuclear magnetization for MRI and radiographic density for CT. We present here our PET-ultrasound phantom and PET-TRUSCT-MRI phantom development, including multi-modality images of phantoms. We also discuss alternative phantom construction options. This phantom development is applicable to many multi-modality applications.

\section{PET-Ultrasound PhANTOM}

We have constructed a simple PET-ultrasound prostate phantom as proof of principle. It was constructed with two basic tissue mimicking materials (TMMs). The "pelvis" was prepared as a high-scatter ultrasound TMM, using $4 \%$ agarose mixed with deionized water and heated to $62{ }^{\circ} \mathrm{C}$ (in a hot water bath on a hot plate and mixed continuously with a magnetic stir rod). The temperature of the agarose-water was kept below $70{ }^{\circ} \mathrm{C}$ to maintain the high-scatter ultrasound properties. The "prostate" was prepared as a low-scatter ultrasound TMM, using $8 \%$ gelatin mixed with deionized water and heated until the gelatin dissolved.

The PET-US phantom was constructed in two stages. We first filled a rectangular plastic box with the $4 \%$ agarose TMM, creating a void with a petrolatum-coated plastic rod in the center. As soon as the rectangular agarose "pelvis" hardened, the rod was removed and we filled the inner cylindrical "prostate" region with the 8\% gelatin TMM. Fig. 1a shows a photograph of the PET-US phantom. At each stage, the TMM was mixed with ${ }^{18} \mathrm{~F}$ radioactivity (potassium fluoride in aqueous solution mixed with a drop of food 
coloring) before placing it in a refrigerator to harden. We used short-lived $\left[{ }^{18} \mathrm{~F}\right]$ Fluoride (110 minutes half-life), since ${ }^{18} \mathrm{~F}$ is readily available from our in-house cyclotron and no longlived radioactive waste were generated by the tests. As a result, the gel hardening time was important due to the ${ }^{18} \mathrm{~F}$ half-life. The "pelvis" gel hardened in 52 minutes (using an ice bath within the refrigerator) and the "prostate" hardened in 31 minutes (without ice bath). The resulting phantom had six times higher $511 \mathrm{keV}$ activity density in the inner cylindrical "prostate" than in the outer rectangular "pelvis."

The phantom was roughly centered in an EXACT HR PET scanner, and PET data were acquired with a 20 minute emission scan in 3D mode and 10 minute transmission scan. At the start of the emission scan, the ${ }^{18} \mathrm{~F}$ activity density was $1.07 \mu \mathrm{Ci} / \mathrm{ml}$ in the cylindrical "prostate" gelatin and 0.17 $\mu \mathrm{Ci} / \mathrm{ml}$ in the rectangular "pelvis" agarose. Image reconstruction was performed with attenuation and scatter correction. Fig. 1b shows a reconstructed coronal PET image of the phantom, as well as a horizontal profile through the "prostate" center. The ${ }^{18} \mathrm{~F}$ activity in the cylindrical "prostate" gelatin is clearly visible within the rectangular "pelvis" background activity, with the expected 6 ("prostate") to 1 ("pelvis") relative activity. The ${ }^{18} \mathrm{~F}$ activity is concentrated almost uniformly within the "prostate," but a small asymmetry is seen due to incomplete ${ }^{18} \mathrm{~F}$ mixing during the gel hardening process (Fig. 1b).

The phantom was then imaged using a $5 \mathrm{MHz}$ external Elektra ultrasound system, as shown in Fig. 1c. The ultrasound image clearly shows the low-scatter cylindrical "prostate" gelatin, which is surrounded by the high-scatter "pelvis" agarose. Thus, we have demonstrated our ability to construct and image a custom PET-ultrasound phantom. However, the phantom's mechanical and ultrasound properties did not have long-term stability, especially at room temperature. For instance, this phantom (without preservative) was invaded by fungus and bacteria within about a month even when stored in a refrigerator.

\section{PET-TRUS-CT-MRI PHANTOMS}

Methods for ultrasound phantom construction are well represented in literature [1-5]. Since the phantom described in Section II did not have long-term stability at room temperature, we needed to develop a different phantom construction process. We therefore constructed a multimodality phantom using tissue mimicking mixtures of agar, gelatin, $\mathrm{CuCl}_{2}-2 \mathrm{H}_{2} \mathrm{O}$, EDTA-tetra $\mathrm{Na}$ Hydrate, $\mathrm{NaCl}$, formalin, Germall-Plus ${ }^{\mathrm{TM}}$, glass beads, $\mathrm{BaSO}_{4}$, and deionized water (Table I). Similar agar-gelatin mixtures were proven to have stable mechanical, ultrasound and MRI properties for at least one year [5]. These agar-gelatin-based tissue mimicking materials were mixed with radioactive solutions (with a drop of food coloring). When developing the procedures for this phantom construction, we used short-lived radioactive $\left[{ }^{18} \mathrm{~F}\right]$ Fluoride and a small amount of non-radioactive $0.5 \mathrm{M}$ $\mathrm{HCl}$. Once the phantom construction procedures were finalized, we used long-lived ${ }^{68} \mathrm{GeCl}_{4}$ radioactivity (271 day half-life) in a $0.5 \mathrm{M} \mathrm{HCl}$ solution to allow repeated PET imaging of the same phantom. $(0.5 \mathrm{M} \mathrm{HCl}$ is used to maintain a stable homogenous ${ }^{68} \mathrm{GeCl}_{4}$ solution.) The main purpose of these custom PET-TRUS-CT-MRI phantoms is to develop radioactive TMMs that both approximate the acoustical properties of patients more accurately and have improved longterm stability at room temperature than those developed in Section II.

We constructed two-region PET-TRUS-CT-MRI phantoms with a "prostate" tapered cylinder within a "pelvis" rectangular cuboid. The "pelvis" has outer dimensions of $15 \mathrm{~cm} \times 15 \mathrm{~cm}$ $x 7.5 \mathrm{~cm}$. The "prostate" is $7 \mathrm{~cm}$ deep with a tapering diameter ranging from 5 to $3 \mathrm{~cm}$. We first filled the "pelvis" cubic container with the "Pelvis TMM" (Table I), creating two voids with petrolatum-coated plastic rods. A small $2.5 \mathrm{~cm}$ diameter cylindrical rod was used to create a hole for the TRUS imaging probe. A larger rod with a tapered end was used to create a void for the "prostate." Once the "pelvis" hardened, we removed both rods. We then filled the tapered cylindrical void with a "Prostate TMM" (Table I), having different multi-modality properties and ${ }^{18} \mathrm{~F}$ or ${ }^{68} \mathrm{Ge}$ activity concentrations than the "pelvis." Both TMMs were hardened at room temperature. Theses phantoms are stored with a thin layer of safflower oil on top to minimize dehydration and shrinkage. Fig. 2 shows a photograph of a custom PETTRUS-CT-MRI phantom.

Table I outlines the dry-weight percentages of the materials used to construct the "Prostate TMM" and "Pelvis TMM" of the custom PET-TRUS-CT-MRI phantoms. For initial tests, only the "Prostate TMM" was mixed with $\left[{ }^{18} \mathrm{~F}\right]$ Fluoride due to the time required for gel hardening. For the final phantom, both TMM regions were mixed with ${ }^{68} \mathrm{GeCl}_{4}$ solution. The primary role of each ingredient is summarized below:

- Agar: concentration set to achieve tissue-like ultrasound properties, such as ultrasound propagation speed. Higher agar concentration also produces shorter longitudinal $\left(\mathrm{T}_{1}\right)$ and transverse $\left(\mathrm{T}_{2}\right)$ MRI relaxation times.

- Gelatin: concentration set for tissue-like ultrasound properties, such as ultrasound propagation speed. Concentration must be roughly the same for "prostate" and "pelvis" regions to avoid changes in volumes due to osmosis.

- $\mathrm{CuCl} \mathrm{Cl}_{2}-2 \mathrm{H}_{2} \mathrm{O}$ and EDTA-tetra $\mathrm{Na}$ Hydrate: EDTA forms chelate with the $\mathrm{Cu}^{2+}$ ions to allow $\mathrm{Cu}^{2+}$ to remain mobile, allowing controlled lowering of the $T_{1}$ MRI relaxation time.

- $\quad \mathrm{NaCl}$ : produces tissue-like MRI coil loading.

- Formalin (37\% formaldehyde): cross-links the gelatin, raising the melting point to $78{ }^{\circ} \mathrm{C}$ where the agar component melts.

- Germall-Plus ${ }^{T M}$ : preservative to prevent fungal and bacterial invasion.

- Glass Beads (20 $\mu \mathrm{m}$ average diameter): increases ultrasound attenuation and backscatter to tissue-like levels. Also shortens $\mathrm{T}_{1}$ and $\mathrm{T}_{2}$ MRI relaxation times. 
- $\mathrm{BaSO}_{4}$ : increases radiographic attenuation for CT imaging.

- ${ }^{18}{ }^{18}$ FJFluoride or ${ }^{68} \mathrm{GeCl}_{4}: 511 \mathrm{keV}$ radioactivity for PET imaging.

The expected ultrasound and MRI properties of the phantom are based on previous work by others [5], since precise measurements of these properties were not needed for our application. The expected ultrasound properties include a propagation speed of about $1534 \mathrm{~m} / \mathrm{s}$, a density of about 1.04 $\mathrm{g} / \mathrm{ml}$, and an attenuation coefficient divided by frequency of about $0.14 \mathrm{~dB} / \mathrm{cm} / \mathrm{MHz}$ for the "Prostate TMM" and 0.38 $\mathrm{dB} / \mathrm{cm} / \mathrm{MHz}$ for the "Pelvis TMM." The MRI $\mathrm{T}_{1}$ relaxation times are expected to be about $494 \mathrm{~ms}$ for the "Prostate TMM" and $423 \mathrm{~ms}$ for the "Pelvis TMM." The MRI $\mathrm{T}_{2}$ relaxation times are expected to be approximately $58 \mathrm{~ms}$. Based on direct region of interest measurements of the CT scans of the phantom, the CT number is expected to be about $50 \mathrm{HU}$ for the "Prostate TMM" and about $140 \mathrm{HU}$ for the "Pelvis TMM."

A custom PET-TRUS-CT-MRI phantom, using ${ }^{18} \mathrm{~F}$ in only the "prostate," was imaged with PET, CT and MRI. Using an EXACT HR PET scanner, PET data were acquired with a 60 minute emission scan in 3D mode and 10 minute transmission scan. At the start of the emission scan, the ${ }^{18} \mathrm{~F}$ activity density was $0.33 \mu \mathrm{Ci} / \mathrm{ml}$ in the prostate. Image reconstruction was performed with attenuation and scatter correction. Fig. 3 shows a reconstructed coronal PET image of the phantom, as well as a horizontal profile through the "prostate" center. The ${ }^{18} \mathrm{~F}$ activity is concentrated almost uniformly within the "prostate," but a small asymmetry is seen due to incomplete ${ }^{18} \mathrm{~F}$ mixing during the gel hardening process.

The phantom was imaged with a Nucletron KV ConeBeam CT scanner (100 keV; $16 \mathrm{mAmps)}$ after the ${ }^{18} \mathrm{~F}$ decayed. Reconstruction was performed with a proprietary SmartScatter algorithm using a Cone-Beam CT wedge filter. Fig. 4 shows a reconstructed coronal $\mathrm{CT}$ image of the phantom with increased radiographic attenuation in the "pelvis" due to the $\mathrm{BaSO}_{4}$

After the ${ }^{18} \mathrm{~F}$ decayed, the phantom was also imaged with an $1.5 \mathrm{~T}$ Avanto Siemens MRI scanner using a head coil with a $\mathrm{T}_{1}$-weighted $2 \mathrm{D}$ spin echo pulse sequence $(\mathrm{TE}=7.8 \mathrm{msec}$; $\mathrm{TR}=500 \mathrm{msec}$; field of view $=230 \mathrm{~mm} \times 230 \mathrm{~mm} \times 3 \mathrm{~mm}$; voxel size $=0.4 \mathrm{~mm} \times 0.4 \mathrm{~mm} \times 3 \mathrm{~mm}$ ). Fig. 5a shows a reconstructed $\mathrm{T}_{1}$-weighted MRI image with a darker "prostate" representing a longer $T_{1}$ compared to the "pelvis." The glass beads (used for ultrasound imaging) shortened the $T_{1}$ in the "pelvis" despite having a lower agar concentration. The phantom was also imaged with a $\mathrm{T}_{2}$-weighted $2 \mathrm{D}$ turbo spin echo pulse sequence $(\mathrm{TE}=89 \mathrm{msec} ; \mathrm{TR}=5590 \mathrm{msec}$; field of view $=230 \mathrm{~mm} \times 230 \mathrm{~mm} \times 3 \mathrm{~mm}$; voxel size $=0.4 \mathrm{~mm} \times$ $0.4 \mathrm{~mm} \times 3 \mathrm{~mm}$ ). Fig. $5 \mathrm{~b}$ shows a reconstructed $\mathrm{T}_{2}$-weighted MRI image with a darker "prostate" representing a shorter $T_{2}$ compared to the "pelvis."

A custom PET-TRUS-CT-MRI phantom using ${ }^{68} \mathrm{GeCl}_{4}$ in a $0.5 \mathrm{M} \mathrm{HCl}$ solution was also constructed. The phantom gel is inside a plastic cubic box with a lid that has a hole for TRUS imaging access. In order to prevent ${ }^{68} \mathrm{Ge}$-gel pieces from escaping during TRUS imaging, a condom is used to seal the hole. (The condom base is fixed around a centering ring on top of the container lid. The condom is held in place using a small washer inside the condom tip and a magnet outside the bottom of the plastic container.) When TRUS imaging the phantom, the TRUS probe is covered by a second condom filled with ultrasound gel and pushed against the phantom to minimize air bubbles. At the time of the phantom construction, the ${ }^{68} \mathrm{Ge}$ activity density was $0.73 \mu \mathrm{Ci} / \mathrm{ml}$ in the "prostate" and $0.12 \mu \mathrm{Ci} / \mathrm{ml}$ in the "pelvis."

This custom phantom was imaged using an EXACT HR PET scanner 30 hours after phantom construction. PET data were acquired with a $3 \mathrm{D}$ emission scan followed by a 10 minute transmission scan. Iterative image reconstruction was performed with attenuation and scatter correction. Fig. 6a shows a reconstructed coronal PET image of the phantom, as well as a horizontal profile through the "prostate" center. The ${ }^{68} \mathrm{Ge}$ activity density was initially six times higher in the "prostate" than the "pelvis." The ${ }^{68}$ Ge activity in the "prostate" is clearly visible within the "pelvis" background. However, the "prostate" radioactivity in the PET image has a blurrier edge in this case (e.g., compared to Fig. 1b), and the relative activity shown in the profile is only 4.8 ("prostate") to 1 ("pelvis"). This is probably due to the initial diffusion of the ${ }^{68} \mathrm{Ge}$ tetrachloride molecules, which is discussed in detail in Section IV. This assumption is supported by the nearly uniform radioactivity seen in the PET image of the previously discussed PET-TRUS-CT-MRI phantom constructed with ${ }^{18} \mathrm{~F}$ (Fig. 3).

The phantom was also imaged with a Hitachi Hi-Vision 5500 digital ultrasound system, using a B mode bi-plane TRUS probe in a linear stepper. Fig. $6 \mathrm{~b}$ shows a transrectal ultrasound image of the phantom with a lower-scatter "prostate" surrounded by a higher-scatter "pelvis."

\section{DISCUSSION}

We intended to use this custom PET-TRUS-CT-MRI phantom repeatedly over a year. However, the ${ }^{68} \mathrm{Ge}$ tetrachloride molecules in the "prostate" diffused into the "pelvis" to become roughly uniformly distributed throughout the phantom in less than 57 days. We believe that the ${ }^{68} \mathrm{Ge}$ tetrachloride molecules were small enough to penetrate the gel pores, slowly reaching an equilibrium in radioactive concentration throughout the "prostate" and "pelvis." We should be able to prevent this ${ }^{68} \mathrm{GeCl}_{4}$ diffusion by using a barrier, such as a female latex condom, between the "prostate" and "pelvis" so the radioactivity instead reaches an uniform equilibrium within the "prostate" and "pelvis" separately. This is similar to the latex condom used to seal the TRUS hole (i.e., to prevent ${ }^{68} \mathrm{GeCl}_{4}$-gel pieces from escaping onto the TRUS probe), which successfully prevented diffusion. No ${ }^{68} \mathrm{GeCl}_{4}$ contamination was measured on either the TRUS probe or the probe-side of the condom that was permanently fixed on the phantom, based on swipe tests that were measured accurately with a well gamma counter.

Other tissue mimicking materials could also be used for PET-US phantom construction. Typical ultrasound TMMs include agar, Zerdine ${ }^{\mathrm{TM}}$, urethanes, epoxies, liquids and natural materials. There are three ultrasound TMMs commercially available: Zerdine ${ }^{\mathrm{TM}}$ from CIRs Inc., condensedmilk-based gel from Gammax RMI, and urethane-rubber-based material from ATS Labs. Alternative PET-US phantom 
construction could utilize radioactive water in condensed milkagar-based mixtures [2] or poly(vinyl alcohol) cryogels [4]. However, ${ }^{68} \mathrm{GeCl}_{4}$ diffusion is still expected for these alternative TMMs (due to the relative size of the ${ }^{68} \mathrm{GeCl}_{4}$ molecule), so a diffusion barrier would still be necessary.

We investigated the construction of a PET-TRUS-CT-MRI prostate phantom with a more realistic geometry, using the agar-gelatin mixtures from Section III. The phantom would have structures simulating the prostate, rectal wall and urethra in a background gel with an opening for the TRUS probe (Fig. 7). The urethra is routinely simulated by filling a tube with ultrasound gel with some air bubbles. Since this PETTRUS prostate phantom would be used only to validate image co-registration, the phantom would not have to exactly mimic the PET and TRUS properties of the prostate region. However, the phantom could be used to validate interventional procedures such a brachytherapy (i.e., radiation therapy where small radiation sources or "seeds" are placed inside or next to the area requiring treatment, such as the prostate).

\section{CONCLUSIONS}

We have successfully developed multi-modality phantoms, including PET-TRUS-CT-MRI phantoms with two regions distinguishable by all four imaging modalities and long-term stability at room temperature. These phantoms had a relatively simple geometry, as their main purpose was to validate image co-registration for PET and TRUS prostate imaging. Longlived ${ }^{68} \mathrm{Ge}$ was used to allow repeated imaging, but the longterm use of the phantom for PET imaging was unsuccessful due to diffusion of the ${ }^{68} \mathrm{GeCl}_{4}$ molecules; thus a diffusion barrier between the "prostate" and "pelvis" is required. Based on repeated imaging results, these phantoms appear to have long-term stability otherwise (i.e., except for the ${ }^{68} \mathrm{GeCl}_{4}$ diffusion). The mechanical properties also appear stable and there are no signs of bacterial or fungal invasion, when stored at room temperature for over ten months.

\section{REFERENCES}

[1] C. L. de Korte, E. I. Cespedes, A. F. W. van der Steen, B. Norder and K. Te Nijenhuis, "Elastic and Acoustic Properties of Vessel Mimicking Material for Elasticity Imaging," Ultrason. Imaging, vol. 19, pp. 112126, 1997.

[2] W. D. D'Souza, E. L. Madsen, O. Unal, K. V. Vigen, G. R. Frank, et al., "Tissue mimicking materials for a multi-imaging modality prostate phantom," Med. Phys., vol. 28, pp. 688-700, 2001.

[3] J. E. Browne, K. V. Ramnarine, A. J. Watson and P. R. Hoskins, "Assessment of the acoustic properties of common tissue-mimicking test phantoms," Ultrasound Med. Biol., vol. 29, pp. 1053-1060, 2003.

[4] K. J. M. Surry, H. J. B. Austin, A. Fenster and T. M. Peters, "Poly(vinyl alcohol) cryogel phantoms for use in ultrasound and MR imaging," Phys Med Biol, vol. 49, pp. 5529-5546, 2004.

[5] E. L. Madsen, M. A. Hobson, S. Hairong, T. Varghese and G. R. Frank, "Tissue-mimicking agar/gelatin material for use in heterogeneous elastography phantoms," Phys. Med. Biol., vol. 50, pp. 5597-5618, 2005. 


\section{Table I.}

Dry-weight percents of the various components in the PET-TRUS-CT-MRI custom phantom. The remaining weight percent is deionized water.

\begin{tabular}{|c|c|c|c|c|c|c|c|c|c|}
\hline & 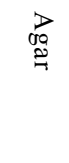 & $\begin{array}{l}\stackrel{Q}{0} \\
\stackrel{0}{\Xi} .\end{array}$ & 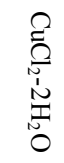 & $\underset{>}{\stackrel{\oplus}{\Xi}}$ & Zֶ, & 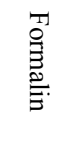 & 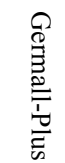 & 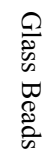 & 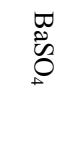 \\
\hline $\begin{array}{l}\text { Pelvis } \\
\text { TMM }\end{array}$ & 1.17 & 5.52 & 0.11 & 0.33 & 0.77 & 0.24 & 1.45 & 4.4 & 0.50 \\
\hline $\begin{array}{c}\text { Prostate } \\
\text { TMM }\end{array}$ & 3.64 & 5.70 & 0.12 & 0.34 & 0.80 & 0.25 & 1.50 & 0 & 0 \\
\hline
\end{tabular}
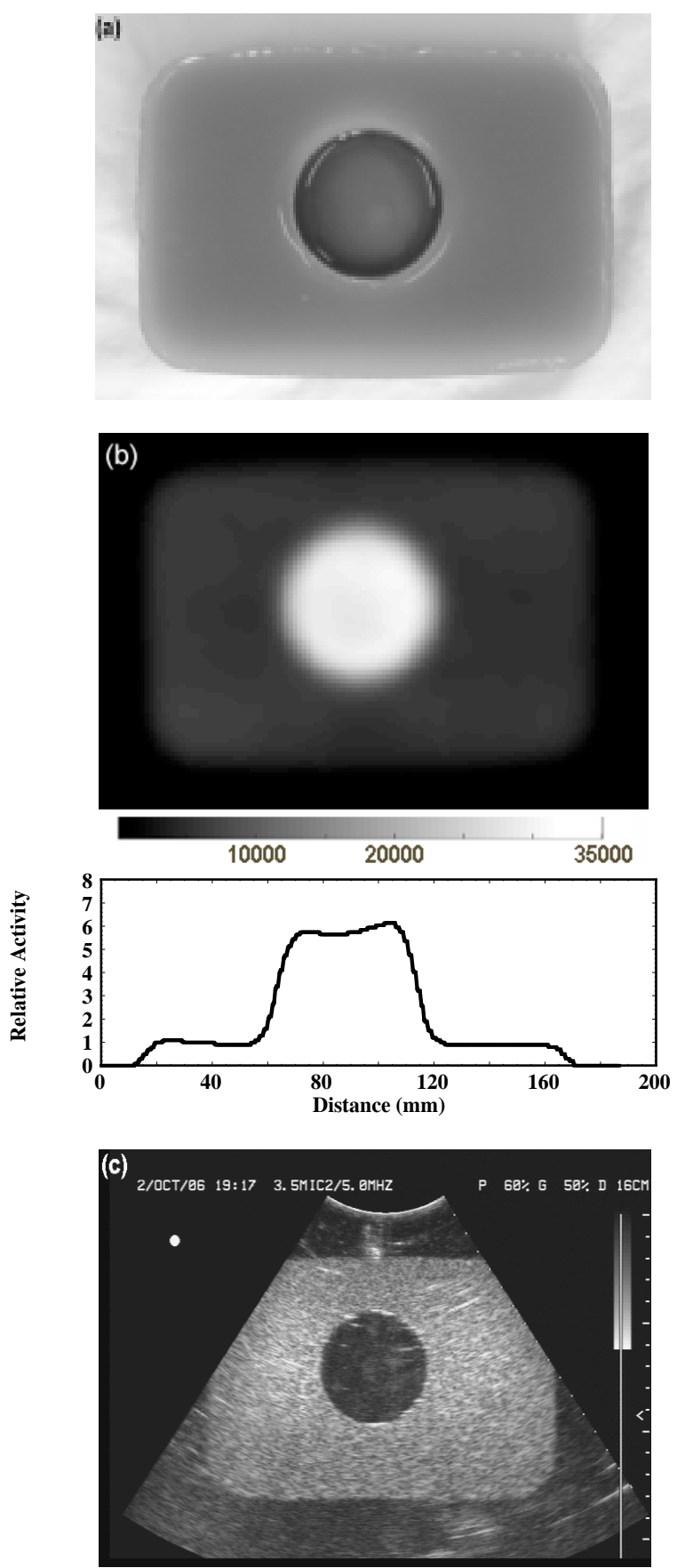

Fig. 1. (a) Photograph of a PET-ultrasound prostate phantom. The agarose "pelvis" has outer dimensions of $16 \mathrm{~cm}$ x $11 \mathrm{~cm} \times 3.5 \mathrm{~cm}$. The cylindrical gelatin "prostate" has a $5 \mathrm{~cm}$ diameter and $2.5 \mathrm{~cm}$ depth. (b) Reconstructed coronal PET image of the phantom. The white circle shows the high ${ }^{18} \mathrm{~F}$ activity density in the "prostate," and the dark gray rectangle shows the low ${ }^{18} \mathrm{~F}$ activity density in the background "pelvis." Image represents $636 \mathrm{M}$ counts (i.e., 20 minutes of data). The voxel size is $1.47 \mathrm{~mm}$ x $1.47 \mathrm{~mm}$ x $3.125 \mathrm{~mm}$. Horizontal profile through the "prostate" center is also shown. (c) Ultrasound image of the same phantom using a $5 \mathrm{MHz}$ external ultrasound probe. The dark gray circle shows the low-scatter gelatin "prostate," and the surrounding light gray background shows the high-scatter agarose "pelvis." 


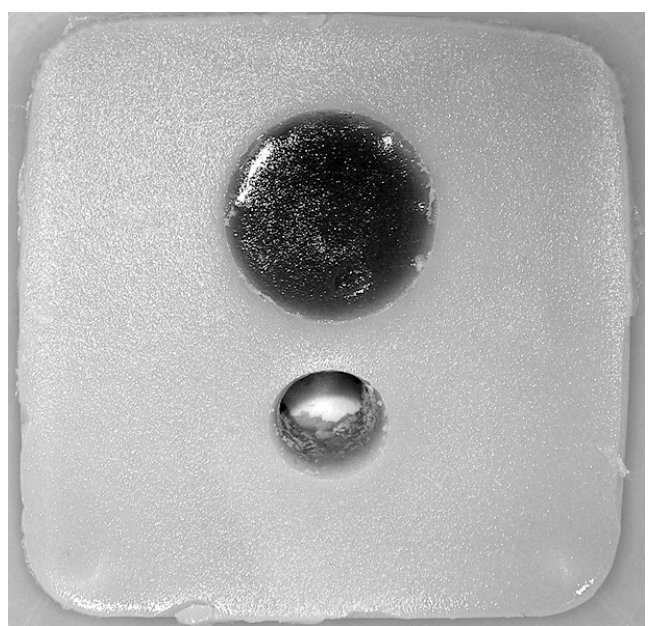

Fig. 2. Photograph of a custom PET-TRUS-CT-MRI phantom. The square "pelvis" has outer dimensions of $15 \mathrm{~cm}$ x $15 \mathrm{~cm}$. The tapered cylindrical "prostate" is seen as a $5 \mathrm{~cm}$ diameter dark circle (the diameter of the cylinder tapers, decreasing from $5 \mathrm{~cm}$ to $3 \mathrm{~cm}$ towards the container bottom). The hole for transrectal ultrasound imaging (seen directly below the dark "prostate" circle) has a diameter of $2.5 \mathrm{~cm}$.
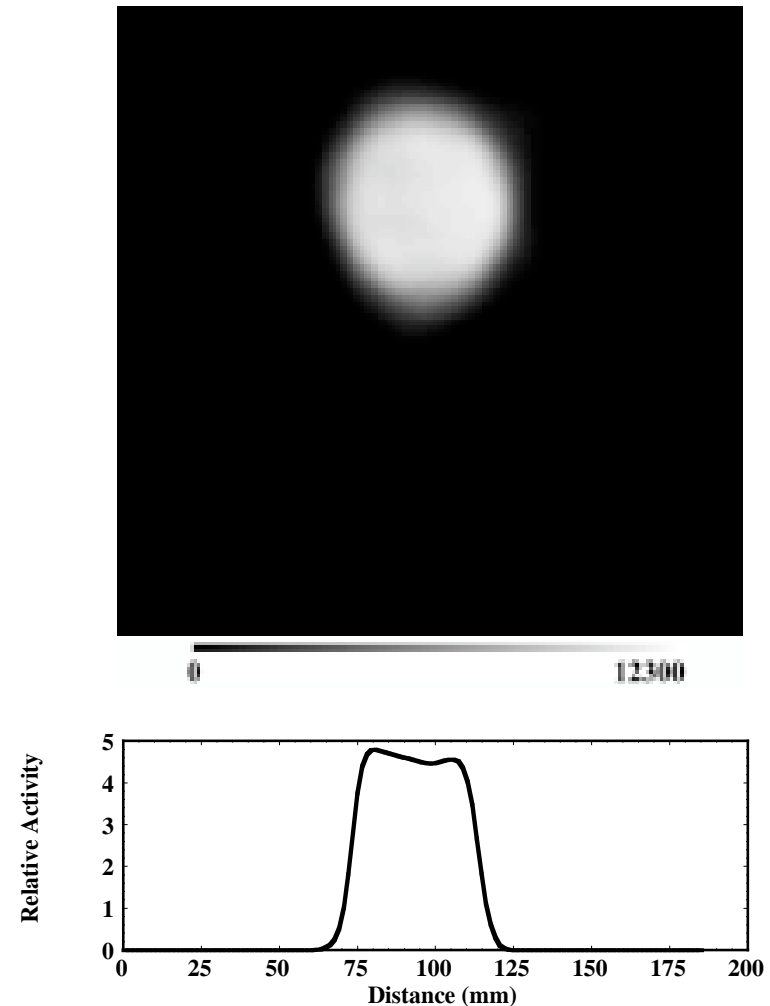

Fig. 3. Reconstructed coronal PET image of a custom PET-TRUS-CTMRI phantom using ${ }^{18} \mathrm{~F}$ (in the "prostate" only). The white circle shows the nearly uniform ${ }^{18} \mathrm{~F}$ activity in the "prostate." The ${ }^{18} \mathrm{~F}$ initial activity density was $0.33 \mu \mathrm{Ci} / \mathrm{ml}$. Voxel size is $1.47 \mathrm{~mm}$ x $1.47 \mathrm{~mm}$ x $3.125 \mathrm{~mm}$. Horizontal profile through the "prostate" center is also shown.

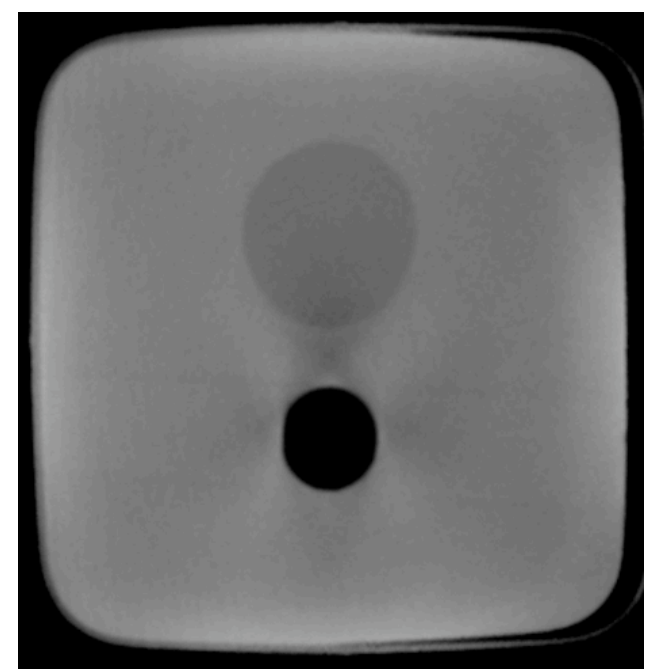

Fig. 4. Reconstructed coronal $\mathrm{x}$-ray CT image of the phantom with $1 \mathrm{~mm}$ axial thick slices. The pixel size is $0.5 \mathrm{~mm} \times 0.5 \mathrm{~mm} \times 1 \mathrm{~mm}$. The dark gray circle shows lower radiographic attenuation in the "prostate" compared with the surrounding light gray higher-attenuation "pelvis." The black circle shows a hole used for the TRUS imaging probe.
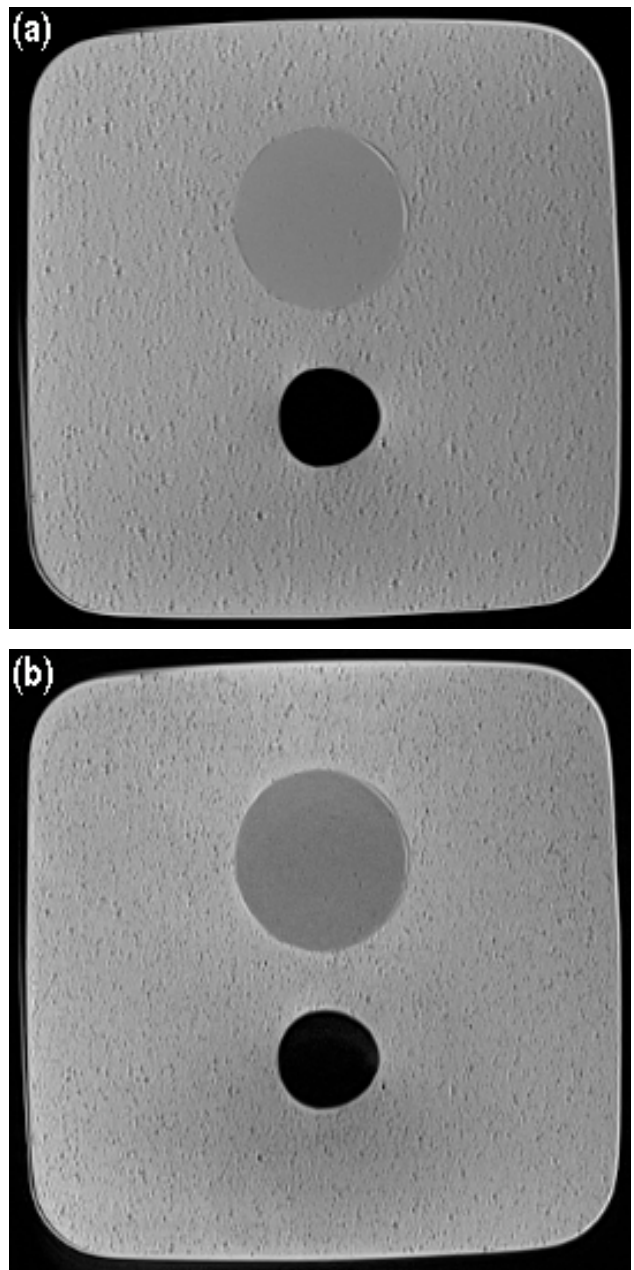

Fig. 5. (a) Reconstructed coronal MRI $\mathrm{T}_{1}$-weighted image of the custom PET-TRUS-CT-MRI phantom, representing 60 minutes of data. Voxel size is $0.4 \mathrm{~mm} \times 0.4 \mathrm{~mm} \times 3 \mathrm{~mm}$. The dark gray circle shows the longer $\mathrm{T}_{1}$ "prostate" surrounded by the shorter $\mathrm{T}_{1}$ "pelvis." The black circle shows a hole used for the TRUS imaging probe. (b) Reconstructed coronal MRI $\mathrm{T}_{2}$-weighted image of the phantom, representing 50 minutes of data. Voxel size is $0.4 \mathrm{~mm} \times 0.4 \mathrm{~mm} \times 3 \mathrm{~mm}$. 

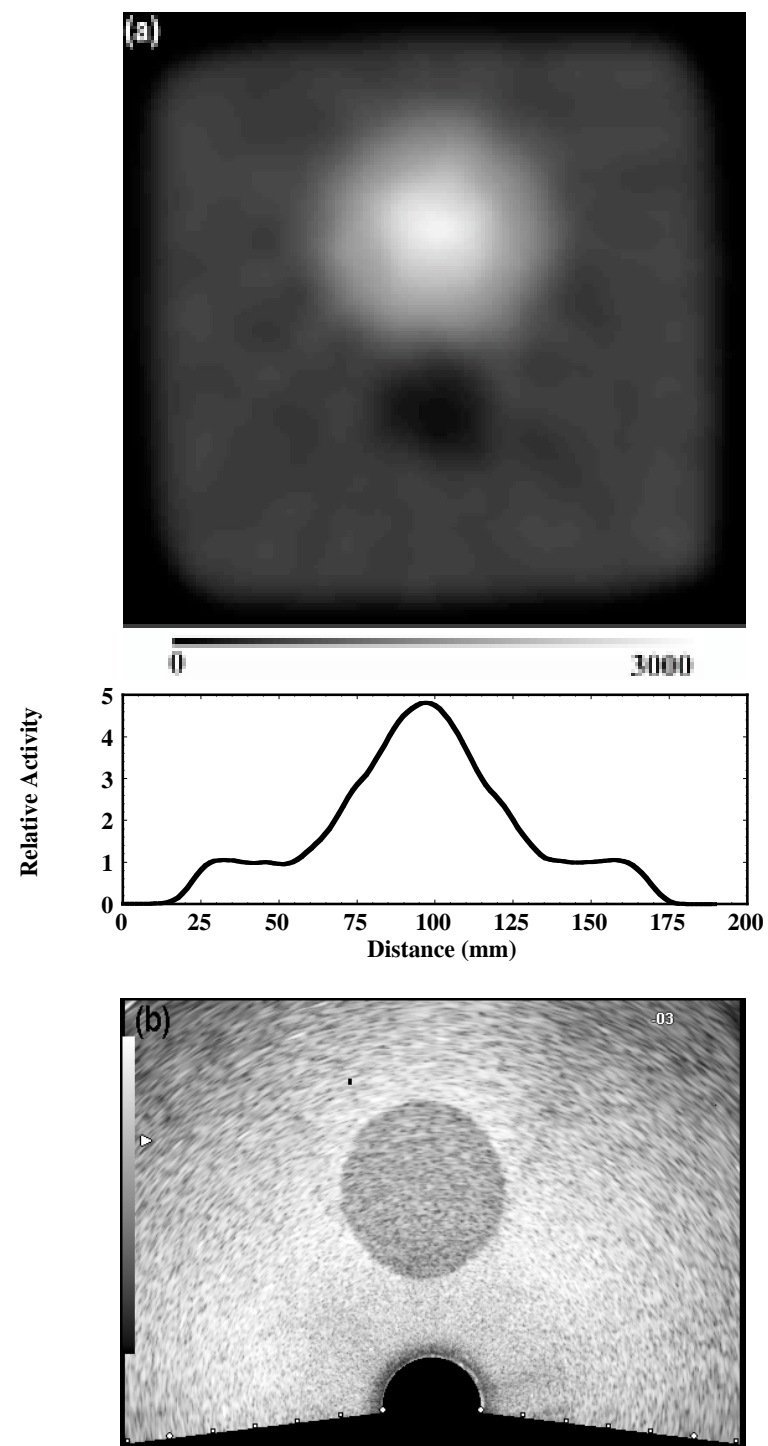

Fig. 6. (a) Reconstructed coronal PET image of a custom PET-TRUS-CTMRI phantom using ${ }^{68} \mathrm{Ge}$. The white circle shows the higher ${ }^{68} \mathrm{Ge}$ activity in the "prostate" within the dark gray low ${ }^{68} \mathrm{Ge}$ activity in the "pelvis." The ${ }^{68} \mathrm{Ge}$ activity density was $0.73 \mu \mathrm{Ci} / \mathrm{ml}$ in the "prostate" and $0.12 \mu \mathrm{Ci} / \mathrm{ml}$ in the "pelvis" during initial construction. Voxel size is $1.47 \mathrm{~mm}$ x $1.47 \mathrm{~mm} \times 3.125$ $\mathrm{mm}$. Horizontal profile through the "prostate" center is also shown. (b) Ultrasound image of the phantom. The dark gray circle shows the lowerscatter "prostate," directly above the black circular hole used for the TRUS probe. The surrounding light gray background shows the higher-scatter "pelvis."
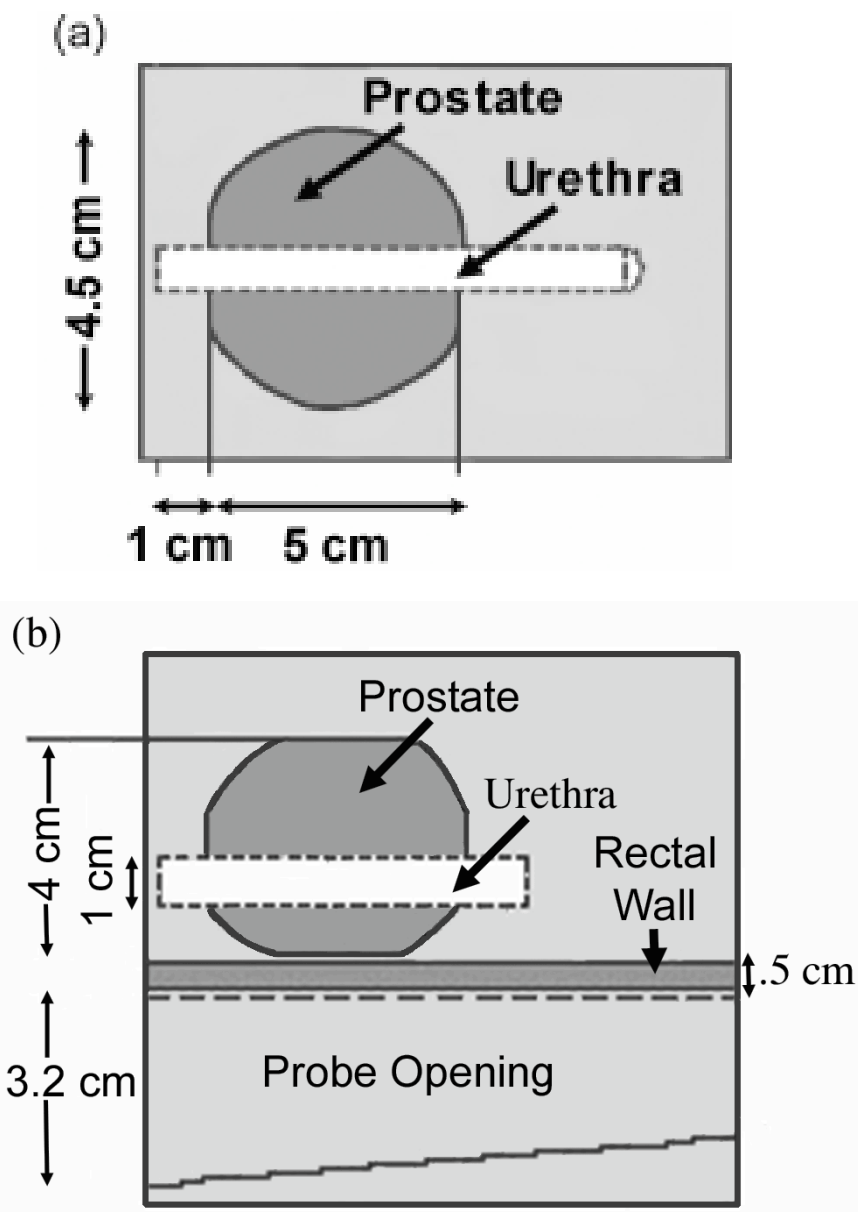

Fig. 7. (a) Top view and (b) side view of design drawing of a PETTRUS-CT-MRI prostate phantom with more realistic geometry. The phantom structures simulate the prostate, rectal wall and urethra in a background gel with an opening for the TRUS probe. 